\title{
Differential sensitivity of various human tumour-derived cell types to apoptosis by organic derivatives of selenium
}

\author{
Raxit J. Jariwalla*†, Bhakti Gangapurkar† and Daniel Nakamura \\ Laboratory of Viral, Immune and Malignant Diseases, California Institute for Medical Research, San Jose, CA 95128, USA \\ (Received 5 March 2008 - Revised 25 April 2008 - Accepted 28 April 2008 - First published online 13 June 2008)
}

Selenium is an important trace element with anti-cancer properties. In the present study, the apoptosis-inducing effects of organic selenium derivatives, namely methyl-L-selenocysteine and selenomethionine, were evaluated in vitro on human tumour-derived cell lines from breast, liver, colon, brain, skin and a non-tumorigenic line of epithelial origin. Apoptosis was assessed by cell-death detection immunoassay on cytoplasmic cell lysates. Breast carcinoma cells were highly sensitive to the organic selenium compounds, manifesting apoptosis at concentrations as low as $0.113 \mu \mathrm{M}(0.0205 \mu \mathrm{g} / \mathrm{ml})$ selenium. By contrast, non-tumorigenic mammary epithelial cells displayed poor sensitivity to selenium, requiring a substantially high concentration of the trace element of $87.9 \mu \mathrm{M}(16.0 \mu \mathrm{g} / \mathrm{ml})$. The cell lines derived from hepatoma and neuroblastoma showed intermediate sensitivity, with colon carcinoma cells manifesting the lowest sensitivity to the trace element. These results indicate intrinsic differences in the sensitivity of human tumour derivatives to selenium-mediated apoptosis, providing experimental support for the development of organic selenium compounds as anti-neoplastic agents against solid tumours displaying selective apoptotic sensitivity to these compounds.

Tumour-derived cells: Cellular apoptosis: Organic selenium derivatives: Methyl-L-selenocysteine: L-Selenomethionine

Selenium is a redox-active trace element with chemopreventive and anti-cancer properties ${ }^{(1,2)}$. It is an integral component of thiol-based antioxidant enzymes such as glutathione peroxidase and thioredoxin reductase, where it occurs in the form of selenocysteine ${ }^{(3)}$. Selenium deficiency has been linked with an increased incidence of certain human cancers $^{(4)}$. Conversely, supplementation with dietary selenium has been shown to reduce the incidence of some human cancers ${ }^{(5,6)}$. Both organic and inorganic compounds of selenium have been demonstrated to manifest cancerpreventive potential, with methylselenocysteine (MSC) displaying optimal potency ${ }^{(1,2,7)}$.

The sensitivity to cancer prevention by selenium varies from tumour to tumour ${ }^{(1,2)}$. Prostrate and lung cancers have shown the strongest link between selenium and cancer prevention ${ }^{(1,2)}$. The reason for only certain cancers being susceptible to protection or inhibition by selenium is not known. One possibility could be the genetic predisposition associated with a particular cell type or cancer. Several studies have shown that tumour cell types lacking the tumour suppressor gene p53 are highly susceptible to chemoprevention by selenium. Among these is breast carcinoma, which is commonly associated with mutations in the p53 gene ${ }^{(8)}$. In addition, other reports have shown that selenium displays chemopreventive properties against cancers of the skin, gastric lesions and other epithelial tumours $(5,6,9)$
Although a large number of chemoprevention studies have been carried out, more recent studies have begun to examine the use of selenium as an anti-cancer agent either alone or in combination with anti-cancer drugs. Studies conducted in tissue culture have shown that high doses of selenium can induce the expression of growth arrest and DNA damage-inducible genes in mouse mammary cells ${ }^{(10)}$. In addition, both inorganic and organic selenium (from yeast) have been shown to induce the death of tumour cells in culture ${ }^{(11-16)}$.

As new therapeutic agents are developed for cancer treatment, it is desirable to evaluate the susceptibility of tumours to compounds with minimal toxicity and superior efficacy. The advantages of utilising organic selenium derivatives are apparent vis-à-vis the toxicity and adverse side effects associated with inorganic selenium compounds. Inorganic forms of selenium such as selenite are metabolised through the hydrogen selenite metabolite pool and produce a spectrum of biological changes that are distinct from organic forms such as MSC that directly enter the methylated pool through methylselenol ${ }^{(3)}$. MSC has distinct advantages as an organic compound, which includes superior in vivo efficacy, non-existent toxicity, low body accumulation and simple formulation $^{(3)}$. Selenomethionine (SeMet) can be converted to selenocysteine through the transsulfuration mechanism ${ }^{(3)}$.

In previous reports on mouse tumour cells, selenite was shown to induce a sequela of cell-growth inhibitory events

Abbreviations: $A_{405 \mathrm{~nm}}$, absorbance at $405 \mathrm{~nm}$; MSC, methylselenocysteine; SeMet, selenomethionine.

* Corresponding author: Raxit J. Jariwalla, fax +1 408588 7107, email r.jariwalla@drrath.com

$\dagger$ Present address: Dr Rath Research Institute, 1260 Memorex Drive, Santa Clara, CA 95050, USA.

\$ Presently at San Bruno, CA, USA. 
including induction of cell death that was accompanied by damage to cellular DNA and loss of cell-membrane integrity ${ }^{(3,13,17)}$. By contrast, MSC and related compounds induced growth inhibition and cell death in the mouse system without producing changes in membrane integrity or cellular DNA as measured by single-strand breaks in DNA ${ }^{(3,18)}$. Further analysis also showed that whereas the predominant type of cell death induced by selenite was necrosis or caspase-independent DNA fragmentation, that induced by MSC was apoptosis or programmed cell death ${ }^{(3,19)}$.

Among the cancer types, prostate, lung and oral carcinomas show enhanced sensitivity to apoptosis by selenium ${ }^{(14-16)}$. The purpose of the present study was to examine the sensitivity to selenium-mediated apoptosis of other major solid tumours of human origin such as those derived from the breast, liver, colon, brain and skin. The main objective was to assess whether such diverse solid tumours manifest similar or differential responses to apoptosis by organic selenium compounds, namely MSC and SeMet. The results obtained suggest that these specific organic derivatives manifest a broad-spectrum anti-cancer activity against diverse tumour cell types that display differential sensitivity to cellular apoptosis by selenium.

\section{Materials and methods}

\section{Cell lines and culture}

The human tumour-derived cell lines and non-tumorigenic epithelial cells evaluated in the present study were obtained from the American Type Culture Collection (Manassas, VA, USA). The cell types used consisted of the following lines of human origin.

Breast cancer cells (MDA-MB-231). A line of adenocarcinoma of the mammary gland derived from pleural effusion at a metastatic site from a 51-year-old Caucasian female. These cells are epithelial in morphology and are tumorigenic in athymic nude mice. The cells were grown in Leibovitz's L-15 medium supplemented with $10 \%$ fetal bovine serum with antibiotics (penicillin and streptomycin) in a humidified atmosphere of $100 \%$ air.

Non-cancerous breast cells (MCF-10A). A line of epithelial morphology derived from the mammary gland of a 36-year-old female with fibrocystic breast disease. The cells are non-tumorigenic in immunosuppressed mice. The cells were grown in a mammary epithelial growth medium (serum free) from Clonetics/BioWhittaker (Walkersville, MD, USA) supplemented with growth factors (SingleQuot additives) and antibiotics (penicillin and streptomycin) in a humidified atmosphere of $5 \% \mathrm{CO}_{2}$ and $95 \%$ air.

Liver adenocarcinoma (hepatoma, SK-HEP-1). A line of epithelial cells derived from the ascitic fluid of a 52-year-old Caucasian male. The cells are tumorigenic in athymic mice. The line was grown in Eagle's minimum essential medium with Earle's balanced salt solution, 2 mM-L-glutamine, nonessential amino acids and sodium pyruvate supplemented with $10 \%$ fetal bovine serum and penicillin plus streptomycin in a humidified atmosphere of $5 \% \mathrm{CO}_{2}$ and $95 \%$ air.

Malignant melanoma (SK-MEL-28). A line of polygonal morphology derived from the skin of a 51-year-old male. The cells form a large round type of malignant melanoma in athymic mice. The cells were grown in the same growth medium and conditions as that used for SK-HEP-1.

Neuroepithelioma (SK-N-MC). A line derived from the metastatic site (supraorbital area) of the brain of a 14-yearold Caucasian female. The cells are tumorigenic in athymic mice and hamster cheek. The cells were cultured in the same growth medium and conditions as those used for SK-HEP-1.

Colon carcinoma (HT-29). A line of colorectal adenocarcinoma of epithelial morphology derived from a 44-year-old Caucasian female. The cells are tumorigenic in athymic mice. The cells were grown in McCoy's 5A medium supplemented with $10 \%$ fetal bovine serum plus penicillin and streptomycin in a humidified atmosphere of $5 \% \mathrm{CO}_{2}$ and $95 \%$ air.

\section{Selenium compounds}

Organic derivatives containing different levels of selenium were obtained from Jarrow Formulas (Los Angeles, CA, USA). The compounds evaluated were as follows: (1) methylselenocysteine (MSC-Sab) and selenomethionine (SeMet-Sab), each containing $0.5 \%$ selenium (in $98.5 \%$ dicalcium phosphate, both originally from Sabinsa Corporation, Piscataway, NJ, USA); (2) a highly purified preparation of methylselenocysteine (MSCGno, containing $43.36 \%$ selenium) from Gnosis S.p.A. (Milan, Italy). Working stock solutions of MSC-Sab and SeMet-Sab were prepared by dissolving the compounds in dilute acid $(0.5 \mathrm{M}-\mathrm{HCl})$ and stocks of MSC-Gno were prepared in sterile water. The working stocks were serially diluted in growth medium before the treatment of cells. Mock solutions (lacking selenium), prepared identically to the selenium stocks, were used for the mock treatment of cells.

\section{In vitro treatments}

For treatment, exponentially growing cells were seeded in ninety-six-well microtitre plates $(100 \mu \mathrm{l}$ of cell suspension per well). After overnight cell attachment at $37^{\circ} \mathrm{C}$, replicate wells of sub-confluent cells were exposed to the respective selenium compound by the direct application of $10 \mu \mathrm{l}$ of selenium-containing solution of appropriate strength. The treatment was repeated once (as applicable) without the change of the growth medium. The actual selenium concentration applied to cells is indicated in Figs. 1-4. Untreated controls received an equivalent amount of mock solution (medium only). After $24-72 \mathrm{~h}$ post-treatment, cells were processed for cellular apoptosis using an enzyme-linked immunoassay, as described in the following section.

\section{Assay of cellular apoptosis}

Apoptosis was quantified using an ELISA (Cell Death Detection ELISA; Roche Applied Sciences, Indianapolis, IN, USA). This immunoassay specifically detects fragmented cellular DNA (histone-oligonucleosomal complexes) in cytoplasmic lysates of test cells. It permits the evaluation of apoptotic cell death in a microtitre plate-based platform following treatment with inducers of apoptosis. Briefly, following the completion of the selenium or mock treatment of target cells, lysates were prepared by the extraction of the treated monolayer with lysis solution followed by low-speed centrifugation $(2500 \mathrm{rpm}, 10 \mathrm{~min})$. The supernatant (cytoplasmic lysate) 
was collected and assayed for the presence of histone-DNA complexes (nucleosomal fragments) by measuring absorbance at $405 \mathrm{~nm}$ in an ELISA plate reader. Apoptosis was assessed in replicate samples and expressed as the mean absorbance at $405 \mathrm{~nm}\left(A_{405 \mathrm{~nm}}\right)$ in the test sample or untreated control.

\section{Data analysis}

Data were analysed by using the software programs Excel and GraphPad Prism. For all selenium formulas, the mean absorbance $\left(A_{405 \mathrm{~nm}}\right)$ values from the cell-death detection ELISA were determined at each concentration level and the fold change relative to the mean of the untreated control (mock treatment) was computed. This relative measure of apoptosis is shown in Figs. 1-4. To estimate the significance of the change, the absorbance values $\left(A_{405 \mathrm{~nm}}\right)$ for each selenium concentration were compared with those of the corresponding untreated control using the paired $t$-test. The means and standard deviations of the $A_{405} \mathrm{~nm}$ values and $P$ values are presented in Tables $1-8$. The $P$ values less than 0.05 were indicative of a statistically significant difference between the treatment and the control means.

\section{Results}

Effect of methylselenocysteine and selenomethionine on breast carcinoma cells

We first examined a human breast carcinoma cell line, MDAMB-231, for its sensitivity to the low (MSC-Sab and SeMet$\mathrm{Sab}$ ) and high (MSC-Gno) selenium-containing derivatives. The effect of the three selenium derivatives on the cellular apoptosis of MDA-MB-231 from a pool of two separate measurements is presented in Fig. 1, which shows the respective fold changes in apoptosis relative to the untreated control. All the three selenium compounds induced the apoptosis of the breast cancer line as evidenced by an increased rate of cell death over the untreated control. The derivatives SeMet-Sab and MSC-Sab produced a striking effect (2-4-fold change over the control), which was detectable at selenium concentrations in the range of $0.105 \mu \mathrm{M}(0.0206 \mu \mathrm{g} / \mathrm{ml})$ to $1.02 \mu \mathrm{M}$ $(0.185 \mu \mathrm{g} / \mathrm{ml})$. SeMet was very effective at the low selenium concentrations tested $(0.105$ and $0.314 \mu \mathrm{M})$, conferring a slightly decreased effect at a higher concentration of the trace element $(0.943 \mu \mathrm{M}=0 \cdot 185 \mu \mathrm{g} / \mathrm{ml}$; Fig. 1 (a)), possibly due to the disruption of the cellular monolayer associated with the low dilutions of the acid-soluble compound. In Fig. 1(b), the water-soluble, selenium-enriched derivative MSC-Gno induced readily detectable apoptosis at a comparable selenium concentration $(9.76 \mu \mathrm{M}=1.777 \mu \mathrm{g} / \mathrm{ml})$, followed by the stabilisation of the effect at higher concentrations of the trace element (Fig. 1(b)). When analysed by the paired $t$-test, the difference between the treatment and control means was significant for all the selenium concentrations tested (Tables 1 and 2).

Effect of methylselenocysteine and selenomethionine on nontumorigenic epithelial cells

To determine whether the apoptosis-inducing effect was specific to tumour-derived breast cells, the sensitivity of a

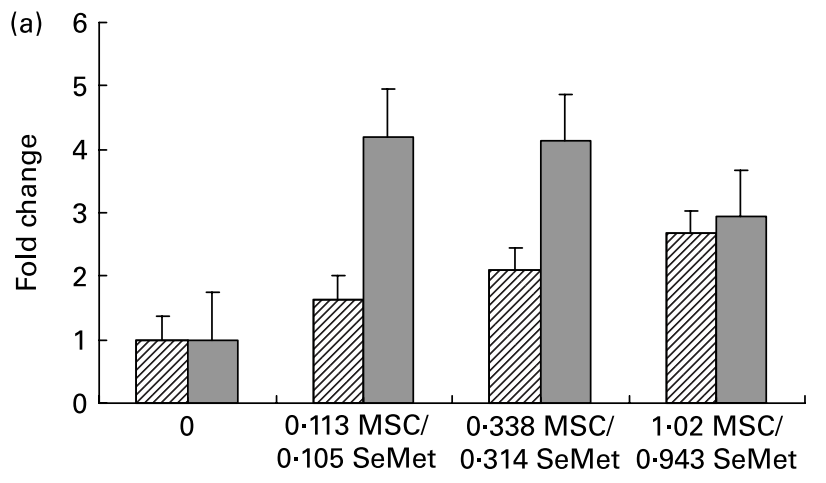

Selenium concentration $(\mu \mathrm{M})$

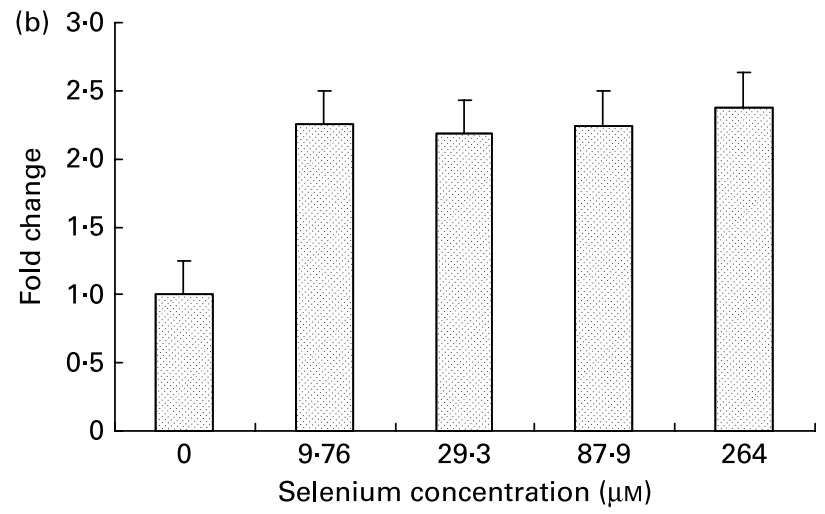

Fig. 1. Cellular apoptosis by the organic selenium compounds (a) MSC-Sab $(\square)$, SeMet-Sab ( $\square$ ) and (b) MSC-Gno (國) in human breast carcinoma cells. Sub-confluent monolayers of MDA-MB-231 cells were incubated with each compound at the indicated concentrations of selenium and, $72 \mathrm{~h}$ later, cytoplasmic cell extracts were assessed for apoptosis by the cell-death detection ELISA, as described in the Materials and methods section. Apoptosis data represent the mean and standard deviation of the fold change in absorbance at $405 \mathrm{~nm}$ relative to the untreated control for quadruplicate determinations. MSC, methylselenocysteine; SeMet, selenomethionine.

non-cancerous line of mammary epithelial cells (MCF-10A) was evaluated using the three selenium-containing derivatives. In contrast to the effect on tumorigenic breast carcinoma cells, the non-cancerous line was resistant to apoptosis by both MSC-Sab and SeMet-Sab, the low selenium-containing derivatives (Fig. 2 (a) and Table 3). The cells were also resistant to moderate concentrations (9.76 and $29.3 \mu \mathrm{M}$-selenium) of MSC-Gno (Fig. 2(b)), which induced insignificant apoptosis $(P>0 \cdot 3)$. The non-tumorigenic cells showed some sensitivity to apoptosis, albeit at only a relatively high concentration $(87.9 \mu \mathrm{M})$ of selenium (Fig. 2 (b) and Table 4).

\section{Sensitivity of human hepatoma cells to methylselenocysteine and selenomethionine}

To determine whether the anti-apoptotic effect of organic selenium derivatives was restricted to a specific type of tumour cells (e.g. breast carcinoma), the sensitivity of SK-HEP-1, a line derived from an adenocarcinoma of the liver (hepatoma), was also evaluated. These cells were readily sensitive to the high selenium-containing derivative MSC-Gno, converting to cells of rounded morphology within $24 \mathrm{~h}$ of treatment (data not shown). By contrast, only a mild effect on cellular 
Table 1. Mean cellular apoptosis by different concentrations of MSC-Sab and SeMet-Sab in human breast cancer cells*

\begin{tabular}{|c|c|c|c|c|c|c|}
\hline \multirow[b]{3}{*}{ Selenium concentration $(\mu \mathrm{M})$} & \multicolumn{3}{|c|}{ MSC-Sab } & \multicolumn{3}{|c|}{ SeMet-Sab } \\
\hline & \multicolumn{2}{|c|}{$A_{405 \mathrm{~nm}}$} & \multirow[b]{2}{*}{$P$ value } & \multicolumn{2}{|c|}{$A_{405 \mathrm{~nm}}$} & \multirow[b]{2}{*}{$P$ value } \\
\hline & Mean & SD & & Mean & SD & \\
\hline 0 (Untreated control) & 0.23 & 0.12 & - & 0.23 & $0 \cdot 12$ & - \\
\hline $0.113 \mathrm{MSC} / 0 \cdot 105 \mathrm{SeMet}$ & 0.37 & 0.07 & 0.0451 & 0.94 & 0.24 & 0.0009 \\
\hline $0.338 \mathrm{MSC} / 0.314$ SeMet & 0.47 & 0.18 & 0.0312 & 0.92 & 0.29 & 0.0022 \\
\hline $1.02 \mathrm{MSC} / 0.943$ SeMet & 0.59 & 0.22 & 0.0140 & 0.66 & 0.36 & 0.0333 \\
\hline
\end{tabular}

MSC, methylselenocysteine; SeMet, selenomethionine; $A_{405 \mathrm{~nm}}$, absorbance at $405 \mathrm{~nm}$.

* Data are mean and standard deviation for quadruplicate determinations of $A_{405 \mathrm{~nm}}$ for fold changes shown in Fig. 1 (a). $P$ values were obtained by comparing absorbance values at $A_{405 \mathrm{~nm}}$ for each selenium concentration with that of the untreated control.

morphology was observed with the two low selenium-containing derivatives MSC-Sab or SeMet-Sab even after $48 \mathrm{~h}$ of treatment, indicating lower sensitivity of this tumour type to selenium compared with that of breast cancer cells.

To quantify the rate of apoptosis, cytoplasmic lysates were harvested from the afore-mentioned cells at 24 or $48 \mathrm{~h}$ post-treatment and assayed by the cell-death detection test. Fig. $3(\mathrm{a}, \mathrm{b})$ shows the sensitivity of the SK-HEP-1 hepatoma cells to the low and high selenium-containing derivatives, respectively, represented as the fold change in apoptosis induced by selenium treatment relative to the untreated control. The results indicated that there was less than 1.3-fold change (Fig. 3 (a)) in apoptosis at $48 \mathrm{~h}$ in the SK-HEP-1 cells exposed to the low selenium-containing derivatives at $0.314-1.02 \mu \mathrm{M}(0 \cdot 0616-0 \cdot 185 \mu \mathrm{g} / \mathrm{ml})$ concentrations ( $P$ values $>0 \cdot 1$ except at an MSC-Sab concentration of $1.02 \mu \mathrm{M}$; Table 5). There was a 2-5-fold increase in apoptosis in the same cells exposed to MSC-Gno at concentrations of 29.3 and $87.9 \mu \mathrm{M}$ ( $P$ values of 0.0122 and $P<0.0001$, respectively; Table 6), consistent with the observations on morphological alteration. In a separate experiment, treatment with a higher concentration of selenium $(264 \mu \mathrm{M}=48.0 \mu \mathrm{g} / \mathrm{ml}$ of MSC-Gno) gave greater than $7 \cdot 0$ fold induction of apoptosis over the untreated control, within $36 \mathrm{~h}$ of treatment (data not shown).

Sensitivity of other tumour cell types (colon carcinoma, neuroepithelioma and melanoma) to methylselenocysteine

To further delineate the apoptosis-inducing effect of MSC-Gno, the compound was examined on other tumour cell types.

Table 2. Mean cellular apoptosis by different concentrations of MSCGno in human breast cancer cells*

\begin{tabular}{llll}
\hline & \multicolumn{3}{c}{ MSC-Gno } \\
\cline { 2 - 3 } & \multicolumn{2}{c}{$A_{405 \mathrm{~nm}}$} \\
\cline { 2 - 3 } Selenium concentration $(\mu \mathrm{M})$ & Mean & SD & $P$ value \\
\hline 0 (Untreated control) & 0.23 & 0.12 & - \\
9.76 & 0.49 & 0.15 & 0.0201 \\
29.3 & 0.51 & 0.20 & 0.0257 \\
87.9 & 0.51 & 0.18 & 0.0221 \\
264 & 0.54 & 0.23 & 0.0295
\end{tabular}

MSC, methylselenocysteine; $A_{405 \mathrm{~nm}}$, absorbance at $405 \mathrm{~nm}$.

* Data are mean and standard deviation for quadruplicate determinations of $A_{405} \mathrm{~nm}$ for fold changes shown in Fig. 1 (b). $P$ values were obtained by comparing absorbance values at $A_{405 \mathrm{~nm}}$ for each selenium concentration with that of the untreated control.
These included a colon cancer line (HT-29) and a line of neuroblastoma cells (SK-N-MC). Sub-confluent monolayers of these cell lines were separately exposed to a range of MSC-Gno concentrations and evaluated for apoptosis. The dose-response effect of the compound on the HT-29 line is shown in Fig. 4 (a) and that on SK-N-MC neuroblastoma cells is presented in Fig. 4(b). The neuroblastoma cells were uniformly sensitive to a wide range of selenium concentrations. Although the fold change was not strikingly high, it was significant at all concentrations tested $(P<0.0005$; Table 8). By contrast, the colon carcinoma cells were affected only by
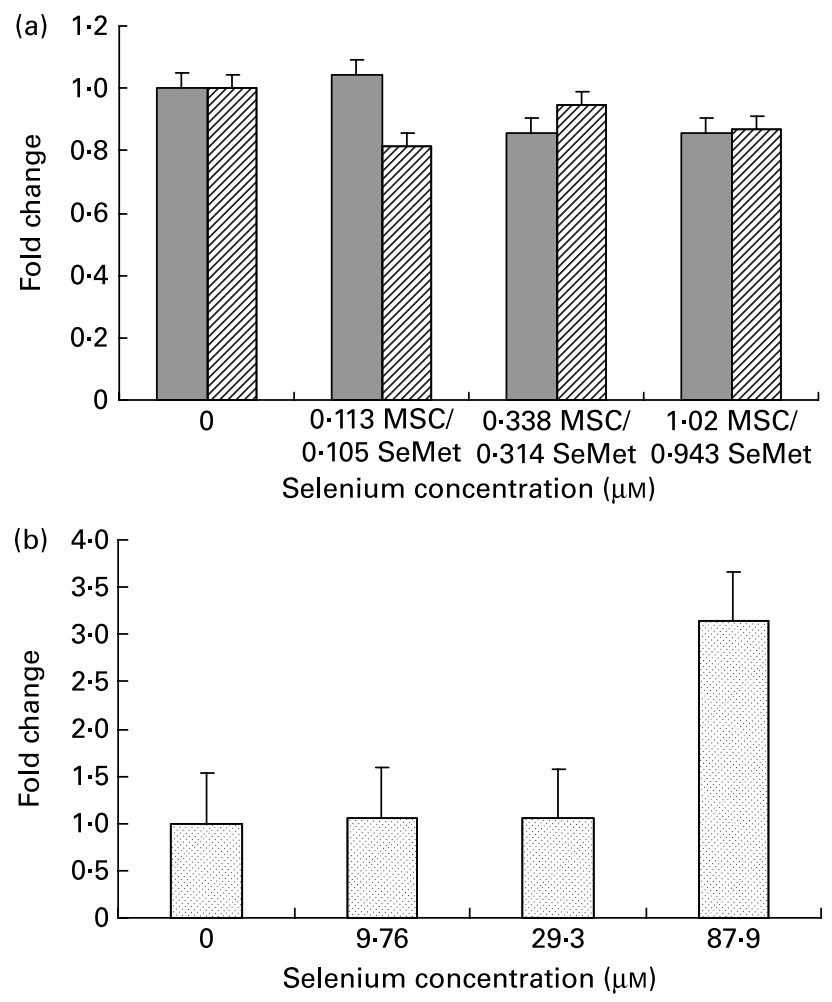

Fig. 2. Effect of the selenium-containing compounds (a) MSC-Sab ( $\square$ ), SeMet-Sab ( $\mathrm{Q}$ ) and (b) MSC-Gno (國) on cellular apoptosis in non-tumorigenic breast epithelial cells. Sub-confluent MCF-10A cells were treated individually with the respective selenium compound and, $72 \mathrm{~h}$ later, cytoplasmic cell extracts were assessed for apoptosis by the cell-death detection assay, as described in the Materials and methods section. Apoptosis data shown are the mean and standard deviation of the fold change in absorbance at $405 \mathrm{~nm}$ relative to the untreated control for triplicate determinations. MSC methylselenocysteine; SeMet, selenomethionine. 
Table 3. Mean cellular apoptosis by different concentrations of MSC-Sab and SeMet-Sab in non-tumorigenic breast epithelial cells*

\begin{tabular}{|c|c|c|c|c|c|c|}
\hline \multirow[b]{3}{*}{ Selenium concentration $(\mu \mathrm{M})$} & \multicolumn{3}{|c|}{ MSC-Sab } & \multicolumn{3}{|c|}{ SeMet-Sab } \\
\hline & \multicolumn{2}{|c|}{$A_{405 \mathrm{~nm}}$} & \multirow[b]{2}{*}{$P$ value } & \multicolumn{2}{|c|}{$A_{405 \mathrm{~nm}}$} & \multirow[b]{2}{*}{$P$ value } \\
\hline & Mean & SD & & Mean & SD & \\
\hline 0 (Untreated control) & 0.35 & 0.07 & - & 0.35 & 0.07 & - \\
\hline $0.113 \mathrm{MSC} / 0.105$ SeMet & 0.37 & 0.36 & 0.467 & 0.29 & 0.03 & 0.0664 \\
\hline $0.338 \mathrm{MSC} / 0.314$ SeMet & 0.30 & 0.10 & 0.217 & 0.33 & 0.13 & 0.403 \\
\hline $1.02 \mathrm{MSC} / 0.943$ SeMet & 0.30 & 0.05 & 0.149 & 0.31 & $0 \cdot 11$ & 0.248 \\
\hline
\end{tabular}

MSC, methylselenocysteine; SeMet, selenomethionine; $A_{405 \mathrm{~nm}}$, absorbance at $405 \mathrm{~nm}$.

* Data are mean and standard deviation for quadruplicate determinations of $A_{405 \mathrm{~nm}}$ for fold changes shown in Fig. 2 (a). $P$ values were obtained by comparing absorbance values at $A_{405 \mathrm{~nm}}$ for each selenium concentration with that of the untreated control.

high concentrations of selenium $(87.9 \mu \mathrm{M}, P$ value $=0 \cdot 0497$ Table 7), showing little or no change at low doses.

A melanoma line (SK-MEL-24) derived from a human tumour was also tested in the present study. Similar to the hepatoma cells, these cells were sensitive at $24-48 \mathrm{~h}$ posttreatment to the high selenium-containing compound (MSCGno, selenium $>27.5 \mu \mathrm{M}$ ), but only poorly sensitive to the low selenium-containing derivatives MSC-Sab and SeMet$\mathrm{Sab}$, as judged by the rounding and shrinking of treated cells, characteristic of apoptosis (data not shown).

\section{Discussion}

In the present study, the apoptosis-inducing effects of organic selenium derivatives, namely MSC and SeMet, were evaluated against various tumour cell types derived from solid cancers and a non-cancerous line of epithelial morphology. The results obtained extend the observations on selenium's anti-cancer action by demonstrating the differential sensitivity of tumour cells to the concentration of the trace element contained in organic selenium derivatives. At the one end of the spectrum were breast cancer cells, which were susceptible to very low concentrations of selenium $(0.113 \mu \mathrm{M}=0.0205 \mu \mathrm{g} / \mathrm{ml})$, whereas at the other end, colon carcinoma cells required substantially higher amounts of the trace element $(>82.4 \mu \mathrm{M})$ for the induction of apoptosis. In the middle of the spectrum, cell lines such as hepatoma and neuroblastoma displayed intermediate sensitivity to the selenium compounds evaluated. In contrast to the apoptotic sensitivity of breast cancer cells

Table 4. Mean cellular apoptosis by different concentrations of MSCGno in non-tumorigenic breast epithelial cells*

\begin{tabular}{llll}
\hline & \multicolumn{3}{c}{ MSC-Gno } \\
\cline { 2 - 3 } & \multicolumn{2}{c}{$A_{405 \mathrm{~nm}}$} \\
\cline { 2 - 3 } Selenium concentration $(\mu \mathrm{M})$ & Mean & SD & $P$ value \\
\hline 0 (Untreated control) & 0.35 & 0.07 & - \\
9.76 & 0.38 & 0.06 & 0.327 \\
29.3 & 0.37 & 0.09 & 0.373 \\
87.9 & 1.11 & 0.20 & 0.0002
\end{tabular}

MSC, methylselenocysteine; $A_{405 \mathrm{~nm}}$, absorbance at $405 \mathrm{~nm}$.

* Data are mean and standard deviation for quadruplicate determinations of $A_{405} \mathrm{~nm}$ for fold changes shown in Fig. 2 (b). $P$ values were obtained by comparing absorbance values at $A_{405 \mathrm{~nm}}$ for each selenium concentration with that of the untreated control.
(MDA-MB-231), the non-cancerous epithelial line MCF-10A was resistant to selenium-mediated apoptosis, indicating the selectivity of the anti-tumour action of selenium. The selenium concentrations found to induce apoptosis of the highly and moderately sensitive tumour lines in the present study are comparable to the plasma selenium levels attained in a high-dose human supplementation trial ${ }^{(20)}$.

Previous studies have evaluated the chemopreventive effect of inorganic derivatives of selenium such as selenite in comparison with that of organic selenium compounds ${ }^{(21,22)}$.
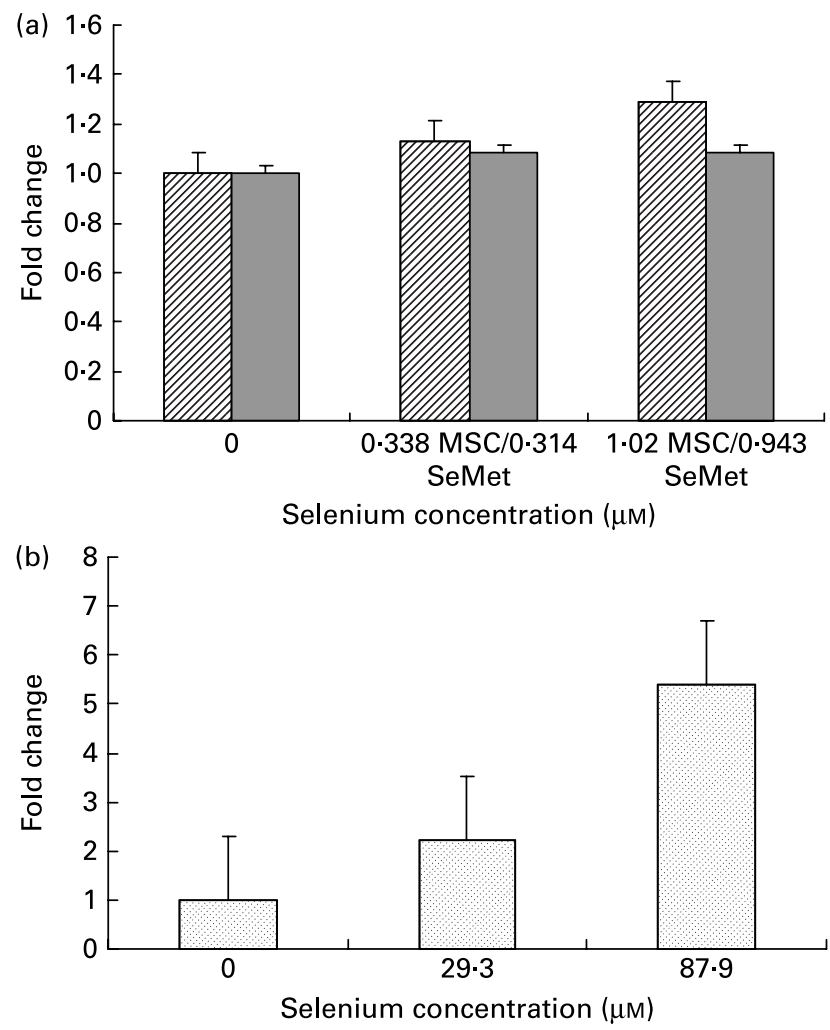

Fig. 3. Cellular apoptosis by the selenium-containing compounds (a) MSCSab ( $\square$ ), SeMet-Sab ( $\square$ ) and (b) MSC-Gno (⿴囗十) in human hepatoma cells. Sub-confluent SK-HEP-1 cells were treated with the respective selenium compound and, 24-48 h later, cytoplasmic cell extracts were assayed for apoptosis, as described in the Materials and methods section. Apoptosis data represent the mean and standard deviation of the fold change in absorbance at $405 \mathrm{~nm}$ relative to the untreated control for triplicate determinations. MSC, methylselenocysteine; SeMet, selenomethionine. 
Table 5. Mean cellular apoptosis by different concentrations of MSC-Sab and SeMet-Sab in human hepatoma cells*

\begin{tabular}{|c|c|c|c|c|c|c|}
\hline \multirow[b]{3}{*}{ Selenium concentration $(\mu \mathrm{M})$} & \multicolumn{3}{|c|}{ MSC-Sab } & \multicolumn{3}{|c|}{ SeMet-Sab } \\
\hline & \multicolumn{2}{|c|}{$A_{405 \mathrm{~nm}}$} & \multirow[b]{2}{*}{$P$ value } & \multicolumn{2}{|c|}{$A_{405 \mathrm{~nm}}$} & \multirow[b]{2}{*}{$P$ value } \\
\hline & Mean & SD & & Mean & SD & \\
\hline 0 (Untreated control) & $1 \cdot 18$ & 0.14 & - & $1 \cdot 18$ & 0.14 & - \\
\hline $0.338 \mathrm{MSC} / 0.314$ SeMet & 1.33 & 0.21 & 0.1785 & 1.28 & 0.09 & 0.1773 \\
\hline 1.02 MSC/0.943 SeMet & 1.52 & 0.03 & 0.0076 & 1.28 & 0.02 & 0.1414 \\
\hline
\end{tabular}

MSC, methylselenocysteine; SeMet, selenomethionine; $A_{405 \mathrm{~nm}}$, absorbance at $405 \mathrm{~nm}$.

*Data are mean and standard deviation for quadruplicate determinations of $A_{405 \mathrm{~nm}}$ for fold changes shown in Fig. 3 (a). $P$ values were obtained by comparing absorbance values at $A_{405 \mathrm{~nm}}$ for each selenium concentration with that of the untreated control.

Other reports have looked at the influence of anti-cancer drugs on human tumour lines in combination with undefined, yeastderived selenium (selenised yeast) that was enriched in SeMet ${ }^{(11)}$. Compared with previous reports, the present investigation was focused on the anti-neoplastic activity of defined organic derivatives of selenium, namely MSC and SeMet, against various cancer cell types derived from solid human tumours.

The advantage of using MSC as a chemopreventive agent over other selenium derivatives has been pointed out by Medina and co-workers ${ }^{(3)}$. The compound directly enters the methylated pool via methylselenol in contrast to inorganic selenium (selenite) that is metabolised through hydrogen selenide. The cells treated with organic selenium compounds that are metabolised via methylselenol were reported to better tolerate selenium and accumulate it to higher levels than sodium selenite $^{(21)}$. In chemopreventive studies conducted in carcinogen-induced rats, compounds such as MSC and selenobetaine (that generate monomethylated selenium) were found to be more efficacious than selenium compounds that are metabolised to hydrogen selenide ${ }^{(23)}$. The results presented here support a selective apoptosis-inducing chemotherapeutic role for both MSC and SeMet against a diverse set of tumour cell types including breast cancer that exhibited maximal sensitivity to the selenium compounds.

The molecular mechanism by which selenium exhibits a selective anti-tumour effect is not known. Both selenoproteins and selenium metabolites have been considered as possible mediators of the anti-cancer action of selenium. Selenium interacts with proteins in two ways ${ }^{(3)}$, which may be of significance to its apoptosis-inducing effect. One interaction involves the incorporation of selenocysteine (in stoichiometric amounts)

Table 6. Mean cellular apoptosis by different concentrations of MSCGno in human hepatoma cells*

\begin{tabular}{lllr}
\hline & \multicolumn{3}{c}{ MSC-Gno } \\
\cline { 2 - 3 } & \multicolumn{2}{c}{$A_{405 \mathrm{~nm}}$} & \\
\cline { 2 - 3 } Selenium concentration $(\mu \mathrm{M})$ & Mean & SD & $P$ value \\
\hline 0 (Untreated control) & 0.39 & 0.05 & - \\
29.3 & 0.87 & 0.22 & 0.0122 \\
87.9 & 2.11 & 0.12 & $<0.0001$
\end{tabular}

MSC, methylselenocysteine; $A_{405} \mathrm{~nm}$, absorbance at $405 \mathrm{~nm}$.

* Data are mean and standard deviation for quadruplicate determinations of $A_{405 \mathrm{~nm}}$ for fold changes shown in Fig. 3 (b). $P$ values were obtained by comparing absorbance values at $A_{405 \mathrm{~nm}}$ for each selenium concentration with that of the untreated control. as an integral amino acid into the structure of selenoproteins such as glutathione peroxidase and thioredoxin reductase. SeMet can also be incorporated into proteins in place of methionine because it readily acylates methionine-transfer RNA. Another way in which selenium interacts with proteins is through direct binding in non-stoichiometric amounts, as indicated by experiments with selenium radioisotopes ${ }^{(24,25)}$.

Certain functions of selenium as an essential trace element are believed to be mediated through the action of selenoproteins. Whether selenoproteins can mediate cellular apoptosis is still a matter of debate. Several critical reviews have discussed
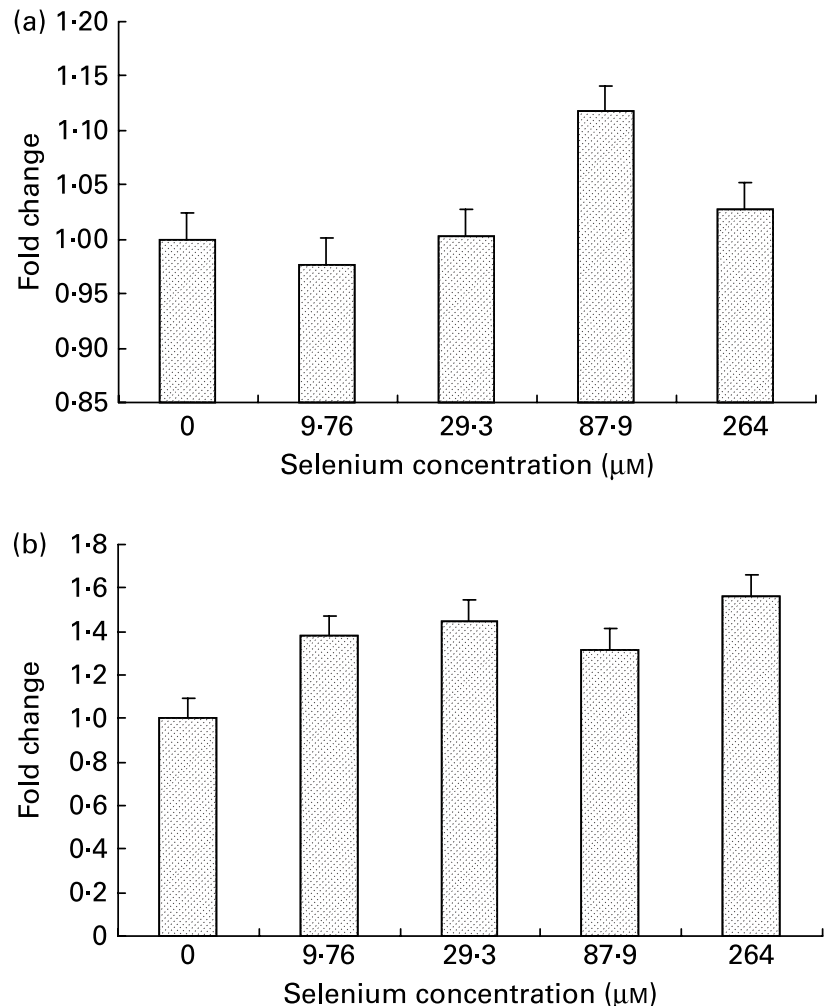

Fig. 4. Effect of MSC-Gno (⿴囗十) on cellular apoptosis in (a) colon carcinoma and (b) neuroblastoma cells. Sub-confluent monolayers of HT-20 (colon carcinoma) and SK-N-MC (neuroblastoma) cells were exposed to MSC-Gno at the indicated selenium concentrations and, after $40 \mathrm{~h}$, cytoplasmic cell extracts were harvested and assayed for apoptosis by the cell-death detection ELISA, as described in the Materials and methods section. Apoptosis data shown are the mean and standard deviation of the fold change in absorbance at $405 \mathrm{~nm}$ relative to the untreated control for septuplicate determinations. MSC, methylselenocysteine. 
Table 7. Mean cellular apoptosis by different concentrations of MSCGno in colon carcinoma cells ${ }^{\star}$

\begin{tabular}{llll}
\hline & \multicolumn{3}{c}{ MSC-Gno } \\
\cline { 2 - 3 } & \multicolumn{2}{c}{$A_{405 \mathrm{~nm}}$} \\
\cline { 2 - 3 } Selenium concentration $(\mu \mathrm{M})$ & Mean & SD & $P$ value \\
\hline 0 (Untreated control) & 2.13 & 0.10 & - \\
9.76 & 2.08 & 0.10 & 0.247 \\
29.3 & 2.13 & 0.03 & 0.457 \\
87.9 & 2.38 & 0.24 & 0.0497 \\
264 & 2.19 & 0.14 & 0.255
\end{tabular}

MSC, methylselenocysteine; $A_{405 \mathrm{~nm}}$, absorbance at $405 \mathrm{~nm}$.

* Data are mean and standard deviation for quadruplicate determinations of $A_{405 \mathrm{~nm}}$ for fold changes shown in Fig. 4 (a). $P$ values were obtained by comparing absorbance values at $A_{405 \mathrm{~nm}}$ for each selenium concentration with that of the untreated control.

the role of selenoproteins in cancer inhibition ${ }^{(2,3,26)}$. Among these, the enzyme glutathione peroxidase, of which selenium is an integral component, has been associated with the protection of cells from oxidative damage. However, in chemopreventive studies, glutathione peroxidase did not appear to be a key mediator of the selenium effect since its enzyme activity, which was already at maximum levels in tissues of animals fed normal amounts of selenium, did not change appreciably as dietary selenium was raised to higher levels required for chemoprevention ${ }^{(26,27)}$.

With regard to thioredoxin reductase, one study showed that the activity of this cytoplasmic enzyme was increased in breast, colon and lung cancer cell lines following supplementation with sodium selenite ${ }^{(28)}$. These data suggested that the elevation of thioredoxin reductase activity might predispose cells to apoptosis. This suggestion was puzzling because thioredoxin and thioredoxin reductase are associated with increased cell growth, proliferation and inhibition of apoptosis $^{(26)}$. To reconcile these disparate observations, Ganther ${ }^{(26)}$ suggested a potential inactivating mechanism for thioredoxin reductase or other selenoenzymes triggered by selenium, which involves the formation of a stable diselenide form resistant to reduction as discussed in the following.

Aside from elemental selenium, its metabolites have also been implicated in the cellular effects of this trace element.

Table 8. Mean cellular apoptosis by different concentrations of MSCGno in neuroblastoma cells*

\begin{tabular}{llll}
\hline & \multicolumn{3}{c}{ MSC-Gno } \\
\cline { 2 - 3 } & \multicolumn{2}{c}{$A_{405 \mathrm{~nm}}$} \\
\cline { 2 - 4 } Selenium concentration $(\mu \mathrm{M})$ & Mean & SD & $P$ value \\
\hline 0 (Untreated control) & 1.64 & 0.14 & - \\
9.76 & 2.08 & 0.21 & 0.0003 \\
29.3 & 2.38 & 0.29 & $<0.0001$ \\
87.9 & 2.13 & 0.18 & $<0.0001$ \\
264 & 2.33 & 0.3 & $<0.0001$
\end{tabular}

MSC, methylselenocysteine; $A_{405 \mathrm{~nm}}$, absorbance at $405 \mathrm{~nm}$.

* Data are mean and standard deviation for quadruplicate determinations of $A_{405 \mathrm{~nm}}$ for fold changes shown in Fig. 4 (b). $P$ values were obtained by comparing absorbance values at $A_{405 \mathrm{~nm}}$ for each selenium concentration with that of the untreated control.
One metabolite class consists of monomethylated forms that are produced from organic selenium derivatives used in the present study. Such metabolites have been proposed to modulate cysteine residues in proteins. The specific mechanism involves selenium as a catalyst of the reversible cysteine/disulfide transformation that occurs in redox-regulated proteins (transcription factors) that may effectively limit the period of time such proteins are in the activated state. According to this concept, protein functions such as redox signalling, catalytic activity or receptor binding could be affected or inactivated by selenium through the formation of such a stable diselenide derivative. This concept is attractive as it does not require a $1: 1$ stoichiometric ratio and is supported by reports showing that enzymes, such as cyclin-dependent kinase 2 and cell-signalling protein kinase, are inhibited by low levels of selenium or its metabolites ${ }^{(26)}$. Whether selenoproteins or selenium metabolites are the bona-fide agents that mediate the apoptosis-inducing activity of selenium remains to be established and further studies of selenium compounds that are biologically unavailable as selenoprotein precursors are needed to provide more insight into the anti-cancer mechanism of selenium action.

In conclusion, the present study has shown that the cell lines derived from solid tumours manifest varying degrees of sensitivity to cellular apoptosis by selenium, with breast carcinoma cells showing maximal sensitivity, followed in decreasing order by hepatoma, neuroepithelioma and colon carcinoma. In view of the potential therapeutic implications of the in vitro data, further studies in experimental animals are warranted to demonstrate whether the observed in vitro cell type-specific differences translate into similar in vivo differences in the apoptotic sensitivity of various tumours to selenium.

\section{Acknowledgements}

We would like to thank Sid Shastri for his stimulating discussions and Sumedha Singh for her technical assistance during the early phase of the project. The present research was supported by a grant from Jarrow Formulas, Inc. R. J. J. designed, conducted and directed the study, and drafted and finalised the paper; B. G. performed experiments, analysed data and contributed to the drafting of the paper; D. N. advised on statistical methods and contributed to data analysis. The authors do not have any conflicts of interest to disclose.

The experiments were approved by the Institutional Biosafety Committee at the California Institute for Medical Research.

\section{References}

1. Fleming J, Ghose A \& Harrison PR (2001) Molecular mechanisms of cancer prevention by selenium compounds. Nutr Cancer 40, 42-49.

2. Ip C, Dong Y \& Ganther HE (2002) New concepts in selenium chemoprevention. Cancer Metastasis Rev 21, 281-289.

3. Medina D, Thompson H, Ganther H \& Ip C (2001) Se-methylselenocysteine: a new compound for chemoprevention of breast cancer. Nutr Cancer 40, 12-17.

4. Ujiie S, Itoh Y \& Kikuchi H (1998) Serum selenium contents and the risk of cancer. Gan To Kagaku Ryoho 25, 1891-1897.

5. Blot WJ, Li JY, Taylor PR, et al. (1993) Nutrition intervention trials in Linxian, China: supplementation with specific vitamin/ 
mineral combinations, cancer incidence, and disease-specific mortality in general population. $J$ Natl Cancer Inst $\mathbf{8 5}$, $1483-1492$.

6. Clark LC, Combs GF Jr, Turnbull BW, et al. (1996) Effects of selenium supplementation for cancer prevention in patients with carcinoma of the skin. A randomized controlled trial. Nutritional Prevention of Cancer Study Group. JAMA 276, 1957-1963.

7. Ip C, el-Bayoumy K, Upadhaya P, Ganther H, Vadhanavikit S \& Thompson H (1994) Comparative effect of inorganic and organic selenocyanate derivatives in mammary cancer chemoprevention. Carcinogenesis 15, 187-192.

8. Lu J, Pei H, Ip C, Lisk DJ, Ganther H \& Thompson HJ (1996) Effect of an aqueous extract of selenium-enriched garlic on in vitro markers and in vivo efficacy in cancer prevention. Carcinogenesis 17, 1903-1907.

9. Gail MH, You WC \& Chang YS (1998) Factorial trial of three interventions to reduce the progression of precancerous gastric lesions in Shangdong, China: design issues and initial data. Control Clin Trials 19, 352-369.

10. Kaeck M, Lu J, Strange R, Ip C, Ganther HE \& Thompson HJ (1997) Differential induction of growth arrest inducible genes by selenium compounds. Biochem Pharmacol 53, 921-926.

11. Vadgama JV, Wu Y, Shen D, Hsia S \& Block J (2000) Effect of selenium in combination with adriamycin or taxol on several different cancer cells. Anticancer Res 20, 1391-1414.

12. Li J, Zuo L, Shen T, Xu CM \& Zhang ZN (2003) Induction of apoptosis by sodium selenite in human acute promyelocytic leukemia NB4 cells: involvement of oxidative stress and mitchondria. J Trace Elem Med Biol 17, 19-26.

13. Zhou N, Xiao H, Li TK, Nur-E-Kamal A \& Liu LF (2003) DNA damage-mediated apoptosis induced by selenium compounds. J Biol Chem 278, 29532-29537.

14. Waters DJ, Shen S, Cooley DM, Bostwick DG, Qian J, Combs GF Jr, Glickman LJ, Oteham C, Schlitller D, Morris JS (2003) Effects of dietary selenium supplementation on DNA damage and apoptosis in canine prostate. J Natl Cancer Inst 95, 237-241.

15. Zhong W \& Oberley TD (2001) Redox-mediated effects of selenium on apoptosis cell cycle in the LNCaP human prostate cancer cell line. Cancer Res 61, 7071-7078.

16. Ghose A, Fleming J, el-Bayoumy K \& Harrison PR (2001) Enhanced sensitivity of human oral carcinomas to induction of apoptosis by selenium compounds: involvement of mitogen-activated protein kinase and Fas pathways. Cancer Res 61, 7479-7487.
17. Lu J, Kaeck M, Jiang C, Wilson AC \& Thompson HJ (1994) Selenite induction of DNA strand breaks and apoptosis in mouse leukemic L1210 cells. Biochem Pharmacol 47, 1531-1535.

18. Wilson AC, Thompson HJ, Schedin PJ, Gibson NW \& Ganther HE (1992) Effect of methylated forms of selenium on cell viability and the induction of DNA strand breakage. Biochem Pharmacol 43, 1137-1141.

19. Jiang C, Wang Z, Ganther H \& Lu J (2002) Distinct effects of methylseleninic acid versus selenite on apoptosis, cell cycle, and protein kinase pathways in DU145 human prostrate cancer cells. Mol Cancer Ther 1, 1059-1066.

20. Burk RF, Norsworthy BK, Hill KE, Motley AK \& Byrne DW (2006) Effects of chemical form of selenium on plasma biomarkers in a high-dose human supplementation trial. Cancer Epidemiol Biomarkers Prev 15, 804-810.

21. Thompson HJ, Wilson A, Lu J, Singh M, Jiang C, Upadhyaya P, el-Bayoumy K \& Ip C (1994) Comparison of the effects of an organic and inorganic form of selenium on a mammary carcinoma cell line. Carcinogenesis 15, 183-186.

22. Reddy BS, Upadhaya P, Simi B \& Rao CV (1994) Evaluation of organoselenium compounds for potential chemopreventive properties in colon carcinogenesis. Anticancer Res 14, $2509-2514$.

23. Ip C \& Ganther HE (1990) Activity of methylated forms of selenium in cancer prevention. Cancer Res 50, 1206-1211.

24. Behne D, Hilmert H, Schied S, Gessner H \& Elger W (1988) Evidence for specific selenium target tissues and new biologically important selenoproteins. Biochem Biophys Acta 966, $12-21$.

25. Bansal M, Ip C \& Medina D (1991) Levels and ${ }^{75}$ Se labeling of specific proteins as a consequence of dietary selenium concentration in mice and rats. Proc Soc Exp Biol Med 196, 147-154.

26. Ganther HE (1999) Selenium metabolism, selenoproteins and mechanisms of cancer prevention: complexities with thioredoxin reductase. Carcinogenesis 20, 1657-1666.

27. Yang JG, Hill KE \& Burk RF (1989) Dietary selenium intake controls rat plasma selenoprotein P concentration. J Nutr 119, $1010-1012$

28. Gallegos A, Berggren M, Gasdaska JR \& Powis G (1997) Mechanisms of the regulation of thioredoxin reductase activity in cancer cells by the chemopreventive agent selenium. Cancer Res 57, 4965-4970. 